\title{
Transmittance and Reflectance of an Imprinted Cholesteric Elastomer During a Segregation Process
}

\author{
P. Castro-Garay and Jesus Manzanares-Martinez
}

Additional information is available at the end of the chapter

http://dx.doi.org/10.5772/50747

\section{Introduction}

The interest in the separation of enantiomers of organic compounds has been recently increased in the food, medicine and cosmetic industries. As it is well know, the chirality is extremely important for living systems, because most of the biomolecules are chiral ones. For example, glucose, a sugar exists in one conformation; while the opposite enantiomer cannot be used as a food source. Biochemistry uses only one hand and not the other however the reason for this has not been understood, so far. Over half of the organic compounds in drugs are chiral whose different enantiomers have different effects. For instance, Ritalin prevents hyperactivity in children in one enantiomer, whereas it has not effect when the opposite enantiomer is used. Another example is thalidomide: one enantiomer of thailomide can cure morning sickness of pregnant women, whereas the other causes birth defects. The sense of smell is also sensitive to the chirality of the odor molecules. For instance, limonene has an orange smell whereas the opposite enantiomer smells like lemon [1].This exemplifies the fact that separation of molecules by chirality is an important process in biochemistry and in the pharmaceutical industry. In this paper we suggest a method to optically monitoring the chiral sorting of molecules performed by an elastomer liquid crystal.

Liquid crystal elastomers combine the rubbery elastic properties with the liquid crystals (LC) anisotropy [2]. When these stiff molecules are joined to a large and flexible polymer chain, they leave their muddy liquid macroscopic form and acquire the appearance of a rubbery network. Particularly, imprinted cholesteric elastomers acquire its chirality from an induced chiral structure instead of chiral molecules. Upon cross-linking nematic polymers in a chiral solvent to form a gel, the solvent, which causes a natural twist to the nematic is thus removed but nonetheless leaves behind an imprinted cholesteric elastomer [3]. A spectacular 
property of imprinted networks is their capacity to preferentially absorb and retain right or left molecules from a racemic solvent which renders them chiral pumps. Moreover the elastomers are photonic materials in the sense that exhibit an optical band structure just as the one shown by electrons in metals and semiconductors. Elastomers provide a large spectrum of applications that exploit the possibility of externally handle a band structure by various types of agents like mechanical stress, electric fields chemical solvents for designing devices such as optical sensors, mirrors, laser and filters [2][4].

The purpose of this chapter is to propose an optical method to determine the volume fraction of preferentially absorbed molecules by measuring the transmittance and reflectance spectra during a preferentially absorbing process. Specifically, we are interested in calculate the reflectance and transmittance of circularly polarized light for oblique incidence due to a cholesteric elastomer, assuming arbitrary values of chiral order parameter, $\alpha$. We have thoroughly discussed the solution of the boundary value problem for cholesteric elastomers in sections 4 and have determined the optical spectra of the system in Sec. 5.

\section{Elastic formulation}

A cholesteric elastomer has locally a nematic structure. Consequently, the director vector $\hat{n}=$ $(\cos \phi(z), \sin \phi(z), 0)$ is at $x-y$ plane and $\phi(z)$ is the angle between director vector and $x$ axis (see Fig.1). The director vector has a continuous rotation as function of $z$, which describes a periodic helical structure characterized by a pitch $p$ (or equivalent by wave number $q_{0}=2 \pi / p$ ) [5][6].

The chiral order parameter $\alpha=\xi q_{0}$ is related with the imprinting efficiency of the cholesteric phase, where $\xi=\sqrt{\frac{K_{2}}{D_{1}}}$ is the nematic penetration depth in rubbery networks, $K_{2}$ is the twist elastic constant and $D_{1}$ is local anchoring of the director to the rubbery network. Variations in the chiral order parameter imply changes in the robustness of chiral imprinting, which can be controlled by varying the density of cross-links in the network (affecting $D_{1}$ ) [7].

The energy for an elastomer formed under the presence of a chiral solvent which is subsequently replaced with an achiral one is given by [8],

$$
F=\frac{1}{2} \int\left[K_{2}\left(\frac{d \phi}{d z}\right)^{2}+D_{1} \sin ^{2}\left(\phi-q_{0} z\right)\right] d z .
$$

The Frank free energy to twist the material is given by

$$
F_{f}=\frac{1}{2} K(\hat{n} \cdot \nabla \times \hat{n})^{2}
$$

associated with the first term of the eq. (1)- tend to unwind the cholesteric helix but it finds resistance to rotate in the anchoring of the rubbery network- associate with the second term of eq. (1). $D_{1}$ is proportional to rubbery elasticity modulus and the rubbery elasticity modulus is related with cross-linked density [7]. The scale length or nematic penetration depth rubbery at which the two energy contributions are comparable is usually small $\xi \simeq 10^{-8} \mathrm{~m}$ for a typical $K_{2} \simeq 10^{-11} \mathrm{~J} / \mathrm{m}, D_{1} \simeq \mu(r-1)^{2} / r$, where $r \sim 0.9$ is the anisotropy chain number 


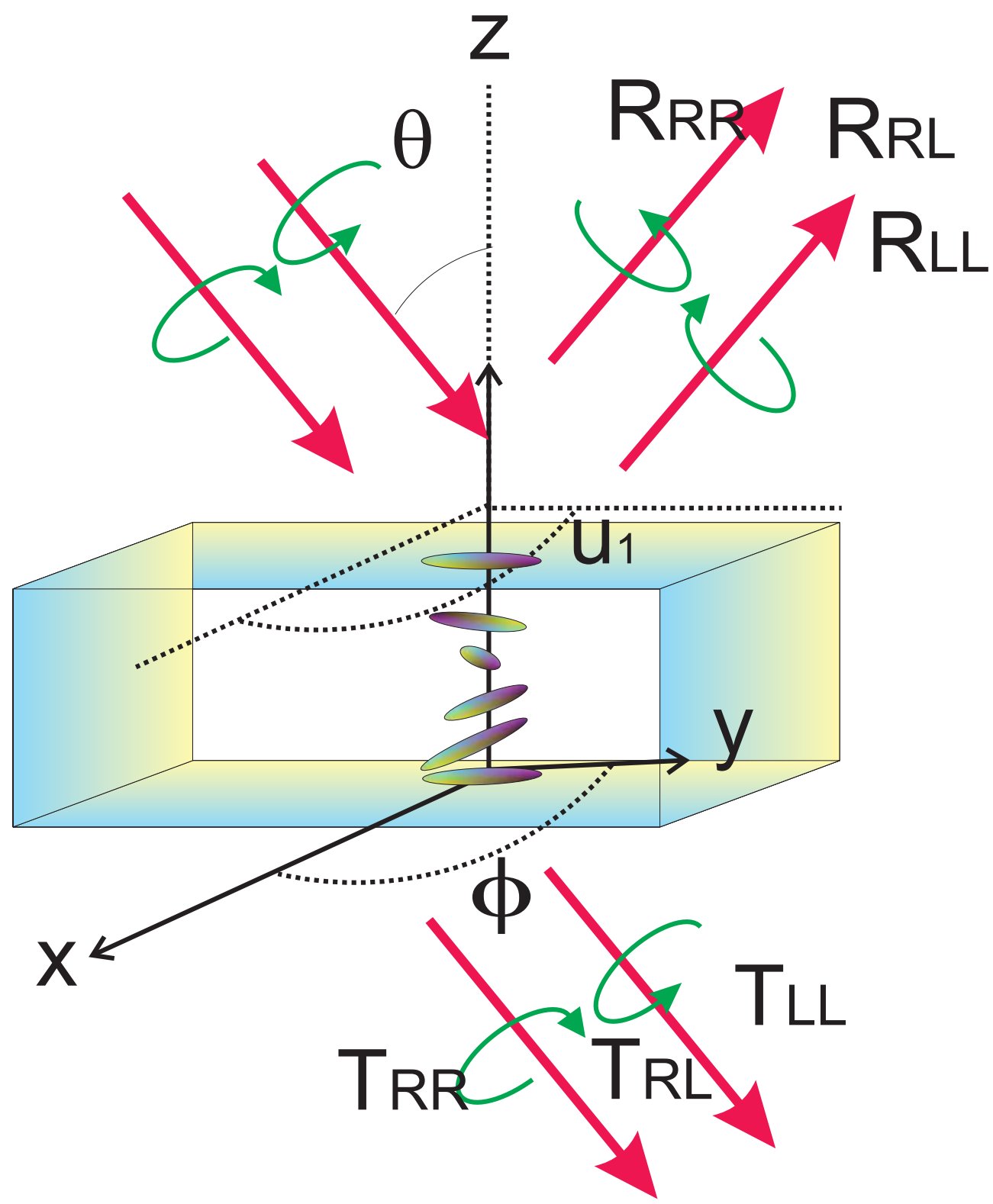

Figure 1. Schematic representation of a liquid crystal cholesteric elastomer. The circular components of the obliquely incident beam as well as the components of the transmitted an reflected parts are shown. 
or cross-linked number and $\mu \simeq 10^{5} \mathrm{~J} / \mathrm{m}^{3}$ is the elasticity rubber modulus. The pitch is $10^{3}$ bigger than nematic penetration depth $\xi$, then

$$
\xi \ll p=\frac{\pi}{q_{0}} .
$$

There are two forms of modifying this relation, i) by increasing the nematic penetration depth which amounts to have a weaker gel or one with smaller anisotropy, ii) by enlarging the Frank free energy. The chiral imprinting will be lost if $D_{1}$ diminish, i.e. so that the director vector anchoring to rubbery will be almost null. Similarly, if the wave number $q_{0}$ or twist elastic constant $K_{2}$ are bigger the chiral imprinting will be also lost. The imprinting efficiency is reached for values of chiral parameter order $\alpha_{c}=\xi q_{o}$ lower than $2 / \pi$.

Eq. (1) can be expressed in terms of chiral parameter order as,

$$
F=\frac{1}{2} \int\left[\frac{\alpha^{2}}{q_{0}^{2}}\left(\frac{d \phi}{d z}\right)^{2}+\sin ^{2}\left(\phi-q_{0} z\right)\right]
$$

The equilibrium configuration can be obtained by minimizing $F$ with respect to the angle, then we have got the Euler-Lagrange equations,

$$
-\partial_{z}\left(\frac{\partial F}{\partial_{z} \phi}\right)=0
$$

which in this case turns out to be

$$
\frac{\alpha^{2}}{q_{0}^{2}} \frac{d^{2} \phi}{d z^{2}}+\sin 2\left(q_{0} z-\phi\right)=0 .
$$

Notice that if $\alpha=0$ the solution of eq. (6) is $\phi_{0}(z)=q_{0} z$ which corresponds to an ideal or undistorted cholesteric helix. By introducing the variable $w=q_{0} z-\phi+\pi / 2$, Eq.(6) transforms in the expression

$$
\frac{d^{2} w}{d z^{2}}+\frac{q_{0}^{2}}{\alpha^{2}} \sin 2 w=0
$$

which is the simple pendulum equation. Hence, for large values of $\alpha$, small harmonic oscillations of frequency $q_{0} / \alpha$ around $\phi=q_{0} z+\pi / 2$ are expected. Nevertheless, for smaller values of $\alpha$ anarmonic oscillations take place until $w$ abandons its oscillatory dependence and grows without limit following the dependence corresponding to a rotatory motion in a simple pendulum. This behavior can be inferred by using the general solution of eq.(6) which is given by

$$
\phi(z)=q_{0} z-A m\left(c z / \xi, 1 / c^{2}\right)+\pi / 2,
$$

where $\operatorname{Am}(z, m)$ is the Jacobian amplitude and $c$ is a constant related with the reduced elastic energy [8] $g=2 \xi F / T D_{1}$. In the Fig. 2 is shown the director vector angle $\phi(z)$ against position for different values of the parameter $c$. In this case, the director vector changes the amplitude 
of its oscillations around the ideal case solution $\phi_{0}(z)$, while the spatial period is maintained constant to increase the values of parameter $c$ from zero until to reach the critical value in $c=1$. Schematic representation of the director vector is shown in the the Fig. 3 . In the Fig. 4 is shown the director vector angle $\phi(z)$ against position for different values of chiral order parameter $\alpha$. It is interesting to note that by increasing $\xi$ from zero, $\phi(z)$ presents oscillations without changing its amplitude, around the undistorted solution $\phi_{0}(z)$ whose spatial period increases, until $\xi$ reaches the critical values: $\xi_{c}=3$. After this value $\phi(z)$ deviate considerably from $\phi_{0}(z)$ and enlarges its effective $q$-value for $\xi \geq \xi_{c}$ which is equivalent to have an untwisted helix than that of $\phi_{0}(z)$ as is shown in the Fig. 5.

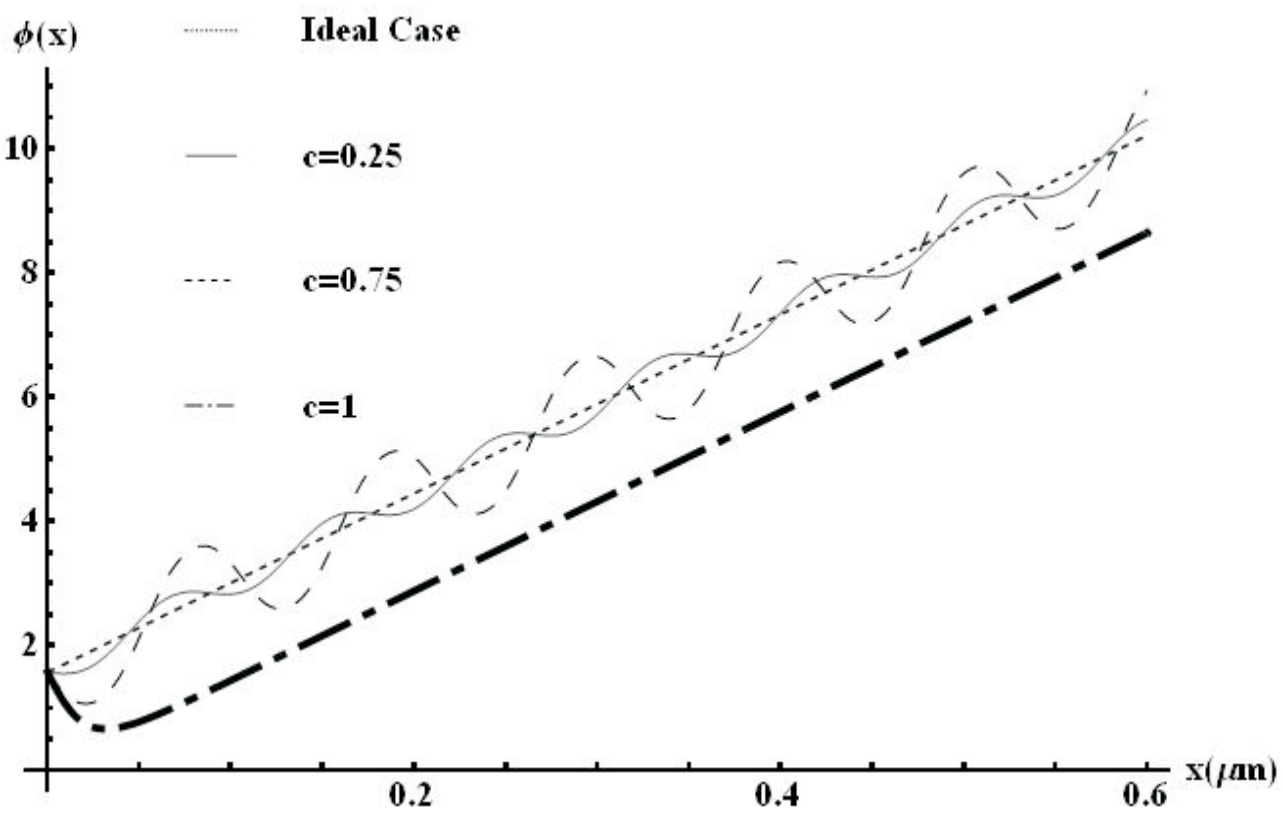

Figure 2. Director vector angle as function of the position for different values of the parameter,c.

\section{Optical description}

As any chiral material whose axis of chiral nonhomogeneity is along the $z$-axis, the optical relative permittivity matrix of the chiral elastomer may be stated as

$$
\bar{\epsilon}(z)=\left(\begin{array}{ccc}
0 & 0 & 0 \\
\epsilon_{\perp}^{(0)}+\epsilon_{a} \cos ^{2} \phi & \epsilon_{a} \sin \phi \cos \phi & 0 \\
\epsilon_{\perp} \sin \phi \cos \phi & \epsilon_{\perp}^{(0)}+\epsilon_{a} \sin ^{2} \phi \epsilon_{\perp}^{(0)}
\end{array}\right)
$$

where $\epsilon_{a}=\epsilon_{\|}-\epsilon_{\perp}$ is the dielectric anisotropy with $\epsilon_{\|}$and $\epsilon_{\perp}$ the dielectric constants in the optical regime parallel and perpendicular to the director $\hat{\mathbf{n}}$, respectively. 


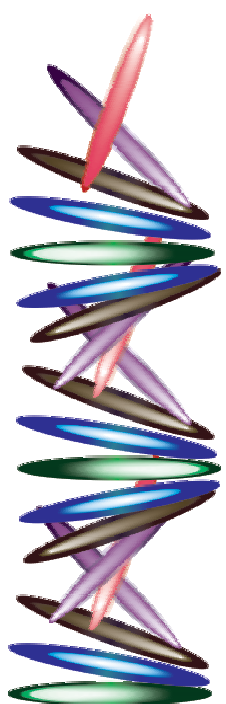

a) Ideal Case

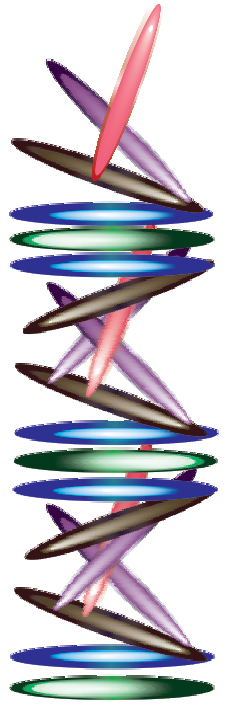

b) $c=0.25$

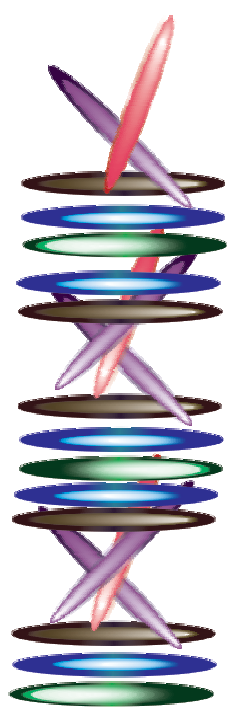

c) $c=0.75$

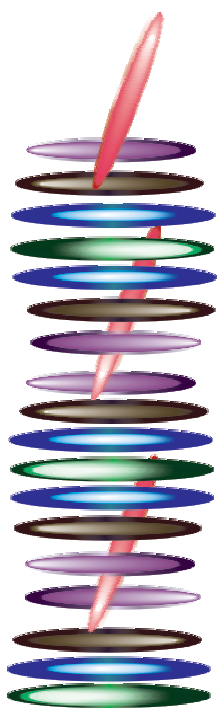

d) $c=0.80$

Figure 3. Schematic representation of director vector for different values of parameter, $c$, a) ideal case, b) $c=0.25$ and $c) c=0.75$.

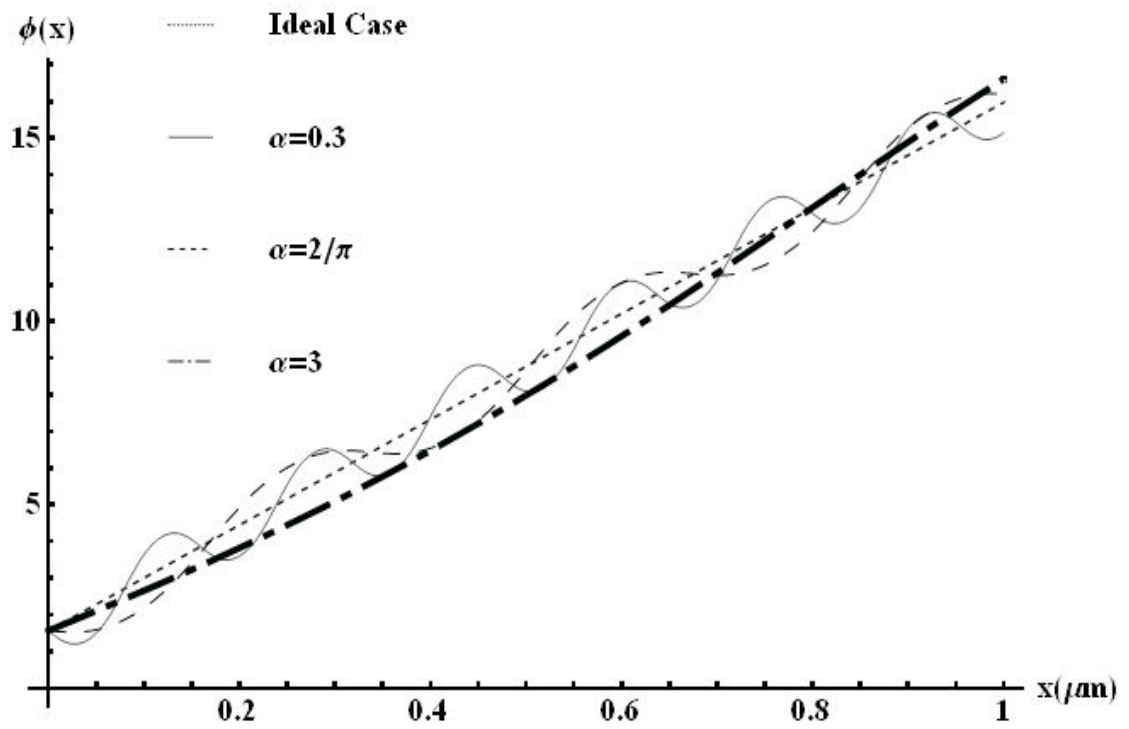

Figure 4. Director vector angle as function of the position for different values of the chiral order parameter, $\alpha$. 

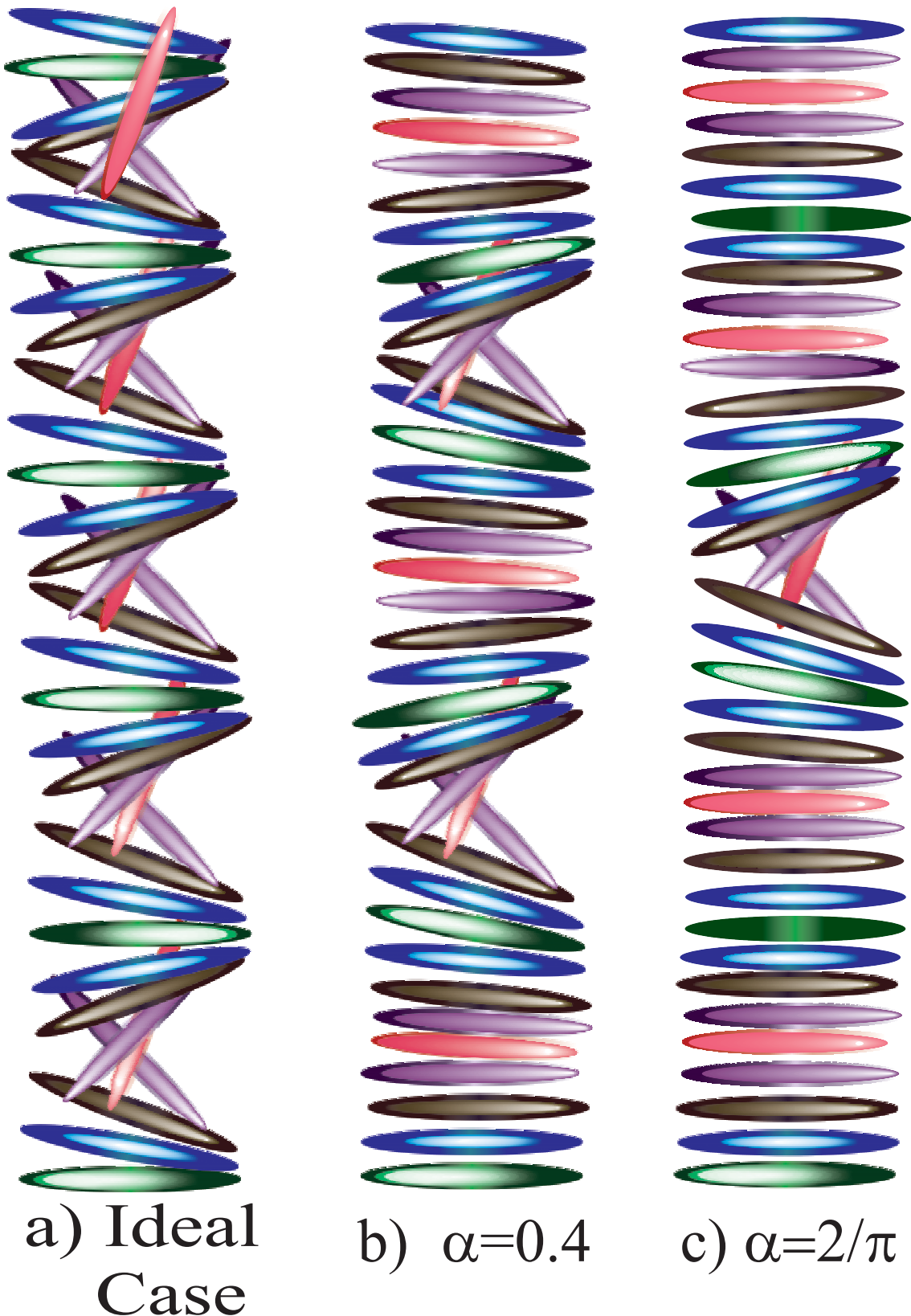

c) $\alpha=2 / \pi$

Figure 5. Schematic representation of director vector for different values of chiral order parameter, $\alpha$, a) ideal case, b) $\alpha=0.3$ and c) $\alpha=2 / \pi$. 
Maxwell curl equations (in MKS units) for media whose dielectric tensor is only $z$-dependent, are given by

$$
\left.\begin{array}{l}
\nabla \times \mathbf{E}(x, y, z)=i \omega \mu_{0} \mathbf{H}(x, y, z) \\
\nabla \times \mathbf{H}(x, y, z)=-i \omega \epsilon_{o} \bar{\epsilon}(z) \cdot \mathbf{E}(x, y, z)
\end{array}\right\}, \quad 0<z<L,
$$

where $\epsilon_{o}$ and $\mu_{0}$ are the permittivity and the permeability of free space (i.e., vacuum). If a plane wave were to be incident obliquely on a Cholesteric Elastomer slab of thickness $L$, we can describe the fields in terms of the column vector [9]

$$
\bar{\psi}(x, y, z)=\exp \left[i \kappa\left(x \cos u_{1}+y \sin u_{1}\right)\right]\left(\begin{array}{c}
e_{x}(z) \\
e_{y}(z) \\
h_{x}(z) \\
h_{y}(z)
\end{array}\right) .
$$

where $u_{1}$ the angle formed by the propagation vector and the $y$-axis in the $x-y$ plane. Thus, the essential part of Maxwell curl equations can then be written as [10].

$$
\frac{d}{d z} \bar{\psi}(x, y, z)=i \bar{A}^{\prime}(z) \cdot \bar{\psi}(x, y, z), \quad 0<z<L,
$$

In this equation, the $4 \times 4$ matrix can be factorized as $\bar{A}^{\prime}=B(z) \cdot \bar{A} \cdot B(-z)$ where the $4 \times 4$ matrix

$$
\begin{aligned}
& \bar{A}(z)=\left(\begin{array}{cccc}
0 & 0 & 0 & \omega \mu_{o} \\
0 & 0 & -\omega \mu_{o} & 0 \\
0 & -\omega \epsilon_{o} \epsilon_{2}^{(0)} & 0 & 0 \\
\omega \epsilon_{o} \epsilon_{d} & 0 & 0 & 0
\end{array}\right)
\end{aligned}
$$

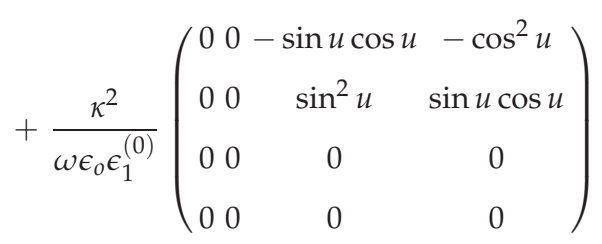

$$
\begin{aligned}
& +\frac{\kappa^{2}}{\omega \mu_{o}}\left(\begin{array}{cccc}
0 & 0 & 0 & 0 \\
0 & 0 & 0 & 0 \\
\sin u \cos u & \cos ^{2} u & 0 & 0 \\
-\sin ^{2} u & -\sin u \cos u & 0 & 0
\end{array}\right)
\end{aligned}
$$

with $u=\frac{h \pi z}{\Omega}-u_{1}$ and 


$$
B(z)=\left(\begin{array}{cccc}
\cos \phi(z) & \sin \phi(z) & 0 & 0 \\
-\sin \phi(z) & \cos \phi(z) & 0 & 0 \\
0 & 0 & \cos \phi(z) & \sin \phi(z) \\
0 & 0 & -\sin \phi(z) \cos \phi(z)
\end{array}\right) .
$$

Equation (12) can be solved by a variety of techniques. We chose to implement the piecewise homogeneity approximation method. The final result is the computation of a transfer matrix $\bar{U}^{\prime}$ such that

$$
\bar{\psi}(L)=\bar{M}^{T}(L) \cdot \bar{U}^{\prime} \cdot \bar{M}(0) \cdot \bar{\psi}(0) \equiv \bar{U} \cdot \bar{\psi}(0) .
$$

\section{Boundary value problem}

Suppose that plane waves are obliquely incident on a cholesteric of finite thickness from both half-spaces $z \leq 0$ and $z \geq L$, which are taken to be vacuous. As a result, the total electric and magnetic fields in these half-spaces may be written as

$$
\left.\begin{array}{c}
\mathbf{e}(z)=\left(a_{L} \hat{\mathbf{u}}_{+}+a_{R} \hat{\mathbf{u}}_{-}\right) \exp \left[i k_{0} z+i \kappa\left(x \cos u_{1}+y \sin u_{1}\right)\right] \\
+\left(r_{L} \hat{\mathbf{u}}_{-}+r_{R} \hat{\mathbf{u}}_{+}\right) \exp \left[-i k_{0} z+i \kappa\left(x \cos u_{1}+y \sin u_{1}\right)\right] \\
\mathbf{h}(z)=i\left(-a_{L} \hat{\mathbf{u}}_{+}+a_{R} \hat{\mathbf{u}}_{-}\right) \exp \left[i k_{0} z+i \kappa\left(x \cos u_{1}+y \sin u_{1}\right)\right] \\
+i\left(-r_{L} \hat{\mathbf{u}}_{-}+r_{R} \hat{\mathbf{u}}_{+}\right) \exp \left[-i k_{0} z+i \kappa\left(x \cos u_{1}+y \sin u_{1}\right)\right] \\
z \leq 0,
\end{array}\right\}
$$

and

$$
\left.\begin{array}{c}
\mathbf{e}(z)=\left(t_{L} \hat{\mathbf{u}}_{+}+t_{R} \hat{\mathbf{u}}_{-}\right) \exp \left[i k_{0}(z-L)+i \kappa\left(x \cos u_{1}+y \sin u_{1}\right)\right] \\
+\left(b_{L} \hat{\mathbf{u}}_{+}+b_{R} \hat{\mathbf{u}}_{-}\right) \exp \left[-i k_{0}(z-L)+i \kappa\left(x \cos u_{1}+y \sin u_{1}\right)\right] \\
\mathbf{h}(z)=i\left(-t_{L} \hat{\mathbf{u}}_{+}+t_{R} \hat{\mathbf{u}}_{-}\right) \exp \left[i k_{0}(z-L)+i \kappa\left(x \cos u_{1}+y \sin u_{1}\right)\right] \\
+i\left(-b_{L} \hat{\mathbf{u}}_{+}+b_{R} \hat{\mathbf{u}}_{-}\right) \exp \left[-i k_{0}(z-L)+i \kappa\left(x \cos u_{1}+y \sin u_{1}\right)\right] \\
z \geq L .
\end{array}\right\}
$$

where

$$
\hat{\mathbf{u}}_{ \pm}=\frac{\hat{\mathbf{u}}_{x} \pm i \hat{\mathbf{u}}_{y}}{\sqrt{2}}
$$

and $\left(\hat{\mathbf{u}}_{x}, \hat{\mathbf{u}}_{y}, \hat{\mathbf{u}}_{z}\right)$ is the triad of Cartesian unit vectors. In these equations, the complex-valued amplitudes $a_{L, R}$ and $b_{L, R}$ of the left- and right-circularly polarized (LCP and RCP) components of incident plane waves are assumed to be known, whereas the reflection amplitudes $r_{R, L}$ and transmission amplitudes $t_{L, R}$ are not known.

Continuity of the tangential components of the electric and the magnetic fields across the planes $z=0$ and $z=L$ leads to the prescriptions of the boundary values 


$$
\bar{\psi}(0)=\bar{Q} \cdot\left(\begin{array}{c}
a_{R} \\
a_{L} \\
r_{R} \\
r_{L}
\end{array}\right), \quad \bar{\psi}(L)=\bar{Q} \cdot\left(\begin{array}{c}
t_{R} \\
t_{L} \\
0 \\
0
\end{array}\right)
$$

where the matrix

$$
\bar{Q}=\left(\begin{array}{cccc}
1 & 1 & 1 & 1 \\
-i & i & i & -i \\
i & -i & i & -i \\
1 & 1 & -1 & -1
\end{array}\right) .
$$

By virtue of Eqs. (16), (17) and (19), we obtain the matrix algebraic equation

$$
\left(\begin{array}{c}
t_{R} \\
t_{L} \\
b_{R} \\
b_{L}
\end{array}\right)=T \cdot\left(\begin{array}{c}
a_{R} \\
a_{L} \\
r_{R} \\
r_{L}
\end{array}\right)
$$

where the so called transfer matrix is given by

$$
T=\frac{1}{4} \bar{Q}^{\dagger} \cdot \bar{U} \cdot \bar{Q}
$$

and the symbol + denotes the conjugate transpose.

The result of solving Eq. (21) is best arranged as

$$
\begin{aligned}
& \left(\begin{array}{c}
t_{R} \\
t_{L} \\
r_{R} \\
r_{L}
\end{array}\right)=S \cdot\left(\begin{array}{l}
a_{R} \\
a_{L} \\
b_{R} \\
b_{L}
\end{array}\right) \\
& S=\left(\begin{array}{cccc}
t_{R R a} & t_{R L a} & t_{R R b} & t_{R L b} \\
r_{L R a} & r_{L L a} & r_{L R b} & r_{L L b} \\
t_{R R a} & t_{R L a} & t_{R R b} & t_{R L b} \\
r_{L R a} & r_{L L a} & r_{L R b} & r_{L L b}
\end{array}\right)
\end{aligned}
$$

where $t_{L R a}$ and $t_{L R b}$, etc., are the transmission coefficients and $r_{L R a}$ and $r_{L R b}$, etc., are the reflection coefficients. The co-polarized transmittances engendered by the waves coming from the left-hand side are denoted by $T_{L L a}=\left|t_{L L a}\right|^{2}$ and $T_{R R a}=\left|t_{R R a}\right|^{2}$, and the cross-polarized ones by $T_{L R a}=\left|t_{L R a}\right|^{2}$ and $T_{R L a}=\left|t_{R L a}\right|^{2}$; and similarly for the reflectances 
$R_{R R a}=\left|r_{R R a}\right|^{2}$, etc. similar definitions are valid for the waves generated by the waves coming from the right-hand side $T_{L L b}=\left|t_{L L b}\right|^{2}, T_{R R b}=\left|t_{R R b}\right|^{2}$ and $T_{L R b}=\left|t_{L R b}\right|^{2}$. The matrix involved $S$ in Eq.(23) is known as the scattering matrix and contains the same information of the transfer matrix but order in different way. It can be straightforwardly shown by writing explicitly the algebraic equations defined by Eq.(21) and solving them for $t_{R}, t_{L}, r_{R}$ and $r_{L}$ that both matrices are related by the expression

$$
S=\left(T \cdot P_{f}-P_{b}\right)^{-1} \cdot\left(P_{f}-T \cdot P_{b}\right),
$$

where $P_{f}$ and $P_{b}$ are the forward and backward projectors given by

$$
P_{f}=\left(\begin{array}{llll}
1 & 0 & 0 & 0 \\
0 & 1 & 0 & 0 \\
0 & 0 & 0 & 0 \\
0 & 0 & 0 & 0
\end{array}\right), P_{b}=\left(\begin{array}{llll}
0 & 0 & 0 & 0 \\
0 & 0 & 0 & 0 \\
0 & 0 & 1 & 0 \\
0 & 0 & 0 & 1
\end{array}\right) \text {. }
$$

In what follows we shall restrict our calculation to the case in which the incident waves are coming only from the left hand side that is we take $b_{R}=b_{L}=0$.

\section{Spectra}

In our calculations we have chosen the physical amounts of a real cholesteric elastomer material $\left(p_{0}=218 \mathrm{~nm}\right)$ [5] [11]. Fig. 6 shows that in the absence of solvent $(\alpha=0)$ there is only a band reflection for right-circularly copolarized light $R_{R R}$ with the bandwidth of $\simeq 50 \mathrm{~nm}$ and maximum amplitude. The center of the reflection band blue-shift whereas the width and amplitude band remain constant as the incidence angle $\theta$ increases. The corresponding reflectances $R_{R L}$ and $R_{L L}$ do no exhibit practically any band. In contrast, when $\alpha=0.4$ there appear bands for both left- and right-copolarized reflectances $R_{R R}$ and $R_{L L}$ whereas the cross-polarized reflectance $R_{R L}$ is almost zero. In this case the $R_{R R}$ is not as well formed as in the absence of solvent because is amplitude present various peaks and the bandwidth varies as a function of the incidence angle, reaching its smaller value for angles around 45 degrees. Differently, $R_{L L}$ is very thin since its bandwidth is around $15 \mathrm{~nm}$ and contained in the interval where the $R_{R R}$ band lies.

Fig. 7 presents the corresponding trasmittances to Fig.6. The case without solvent $(\alpha=$ $0)$ confirms consistently the complemetary behavior of an undistorted cholesteric. The co-polarized $T_{R R}$ and $T_{L L}$ exhibit troughs where the transmittance diminish, the one for $T_{R R}$ is well defined whereas that of $T_{L L}$ changes is thickness a deep. The blue-shift of the trough for large incident angles is also observed. In the Fig. 8 is shown the band reflection for left and right circularly polarized ligh $R_{R R}$ and $R_{L L}$ for different values of parameter, $c$. In both cases, the band reflection for left circularly polarized light is thinner than the band reflection for right circularly polarized light.

In the Fig. 9 is shown the reflectance of left and right circularly polarized light as a function of wavelenght and the chiral order parameter to oblique incidence. Complementary, In the Fig. 10 is shown the same of the Fig.9 as function of wavelenght and the parameter $c$. 

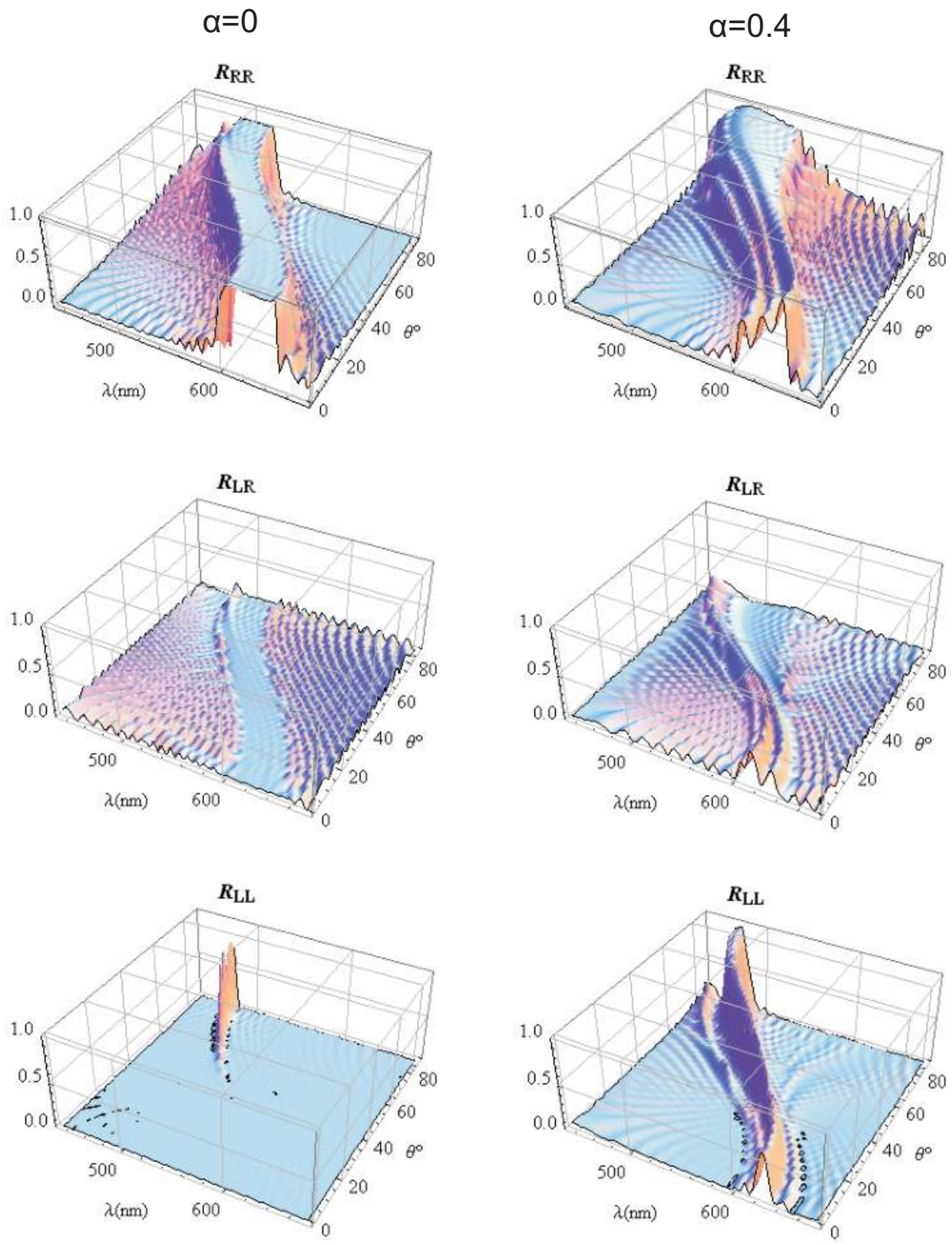

Figure 6. Circularly polarized reflectance $R_{R R}$ and $R_{L L}$ as function of the wavelength and angle incidence for the ideal helix case $\alpha=0$ (left column) and $\alpha=0.4$ (right column). Other parameters are: $\epsilon_{\perp}=1.91, \epsilon_{\|}=2.22, h=1$ and $q_{0}=218 \mathrm{~nm}$. 

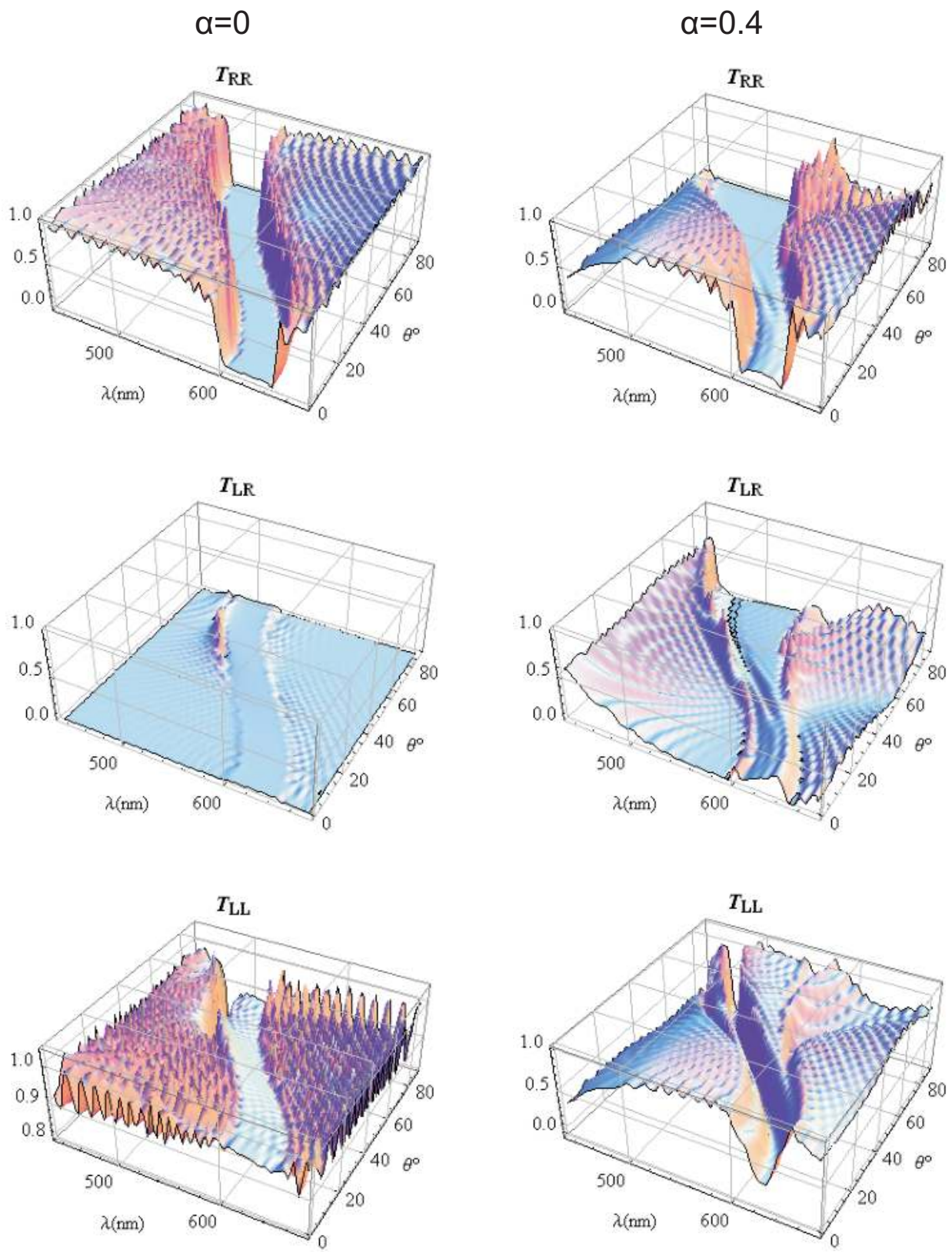

Figure 7. Circularly polarized transmittance $T_{R R}$ and $T_{L L}$ as function of the wavelength and angle incidence for $\alpha=0$ (left column) and $\alpha=0.4$ (right column). 


\section{$c=0.3$}

a)

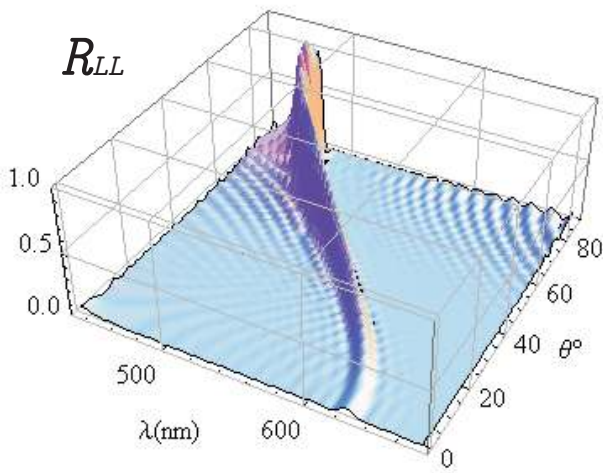

c)

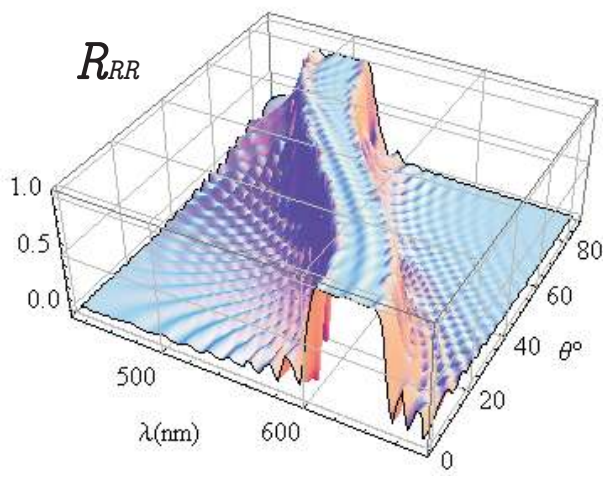

$c=1$

b)

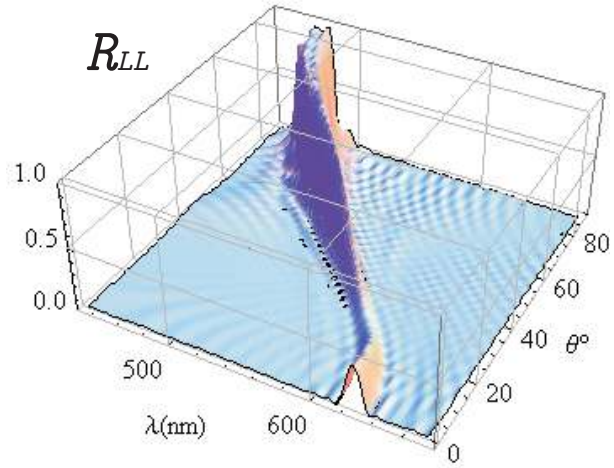

d)

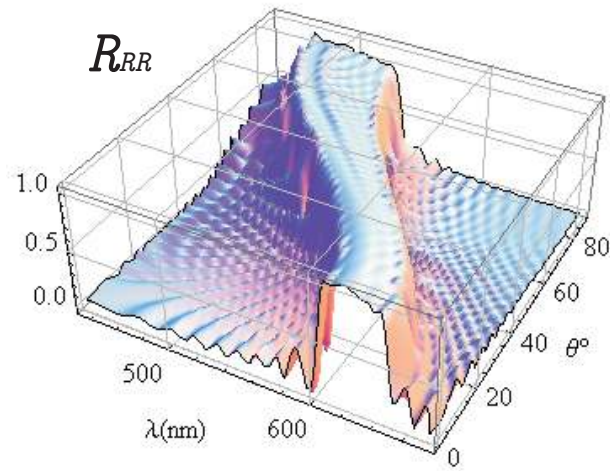

Figure 8. Circularly polarized reflectance $R_{R R}$ and $R_{L L}$ as function of wavelength and angle incidence for $c=0.3$ (left column) and $c=1$ (right column). 
a)

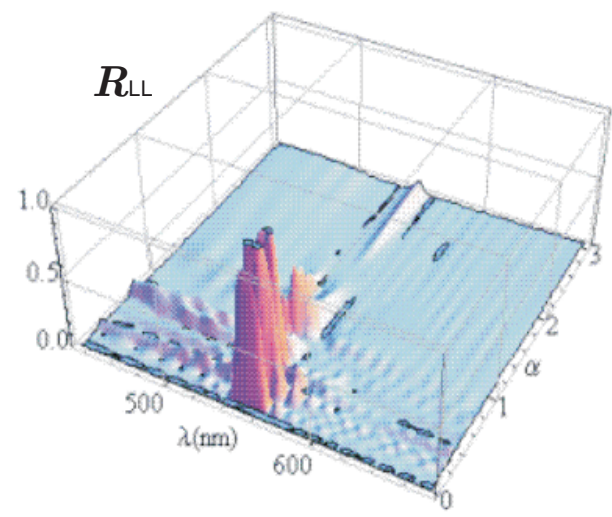

b)

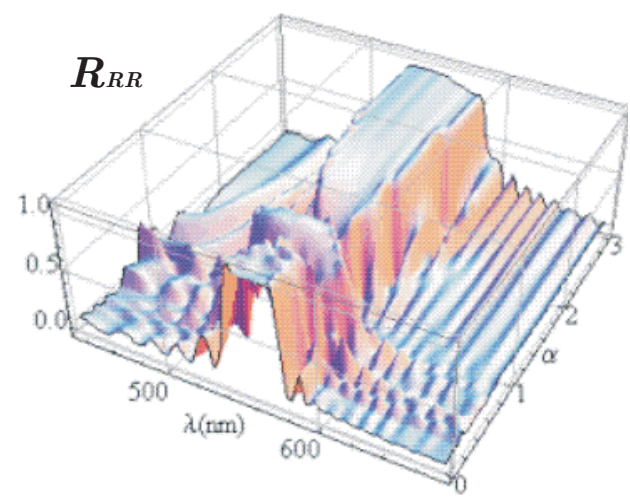

Figure 9. Circularly polarized reflectance $R_{R R}$ and $R_{L L}$ as function of wavelength and the chiral order parameter to oblique incidence angle of $45^{\circ}$ and $c=0.5$.

a)

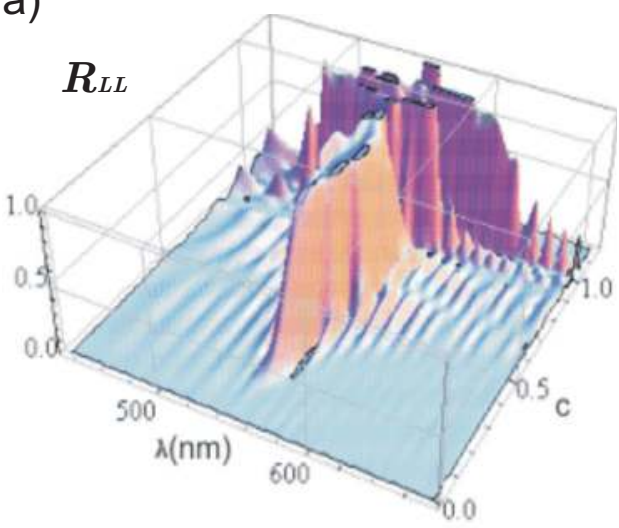

b)

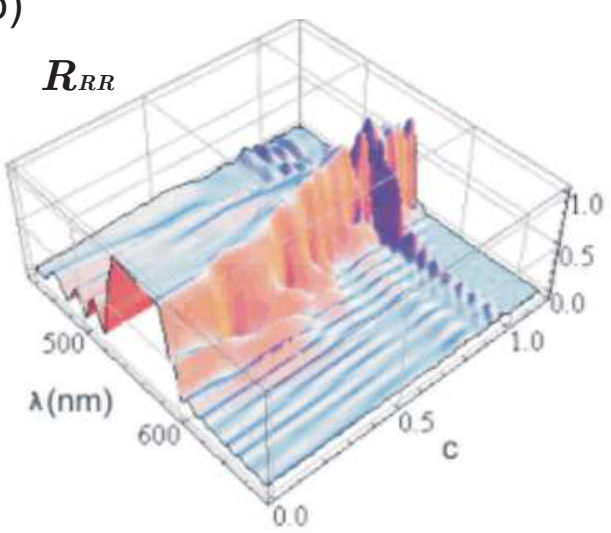

Figure 10. Circularly polarized reflectance $R_{R R}$ and $R_{L L}$ as function of wavelength and the $c$ parameter to oblique incidence angle of $45^{\circ}$ and $\alpha=0.2$. 


\section{Conclusions}

We have obtained the circularly polarized reflectances and transmittances as function of the chiral order parameter of a cholesteric elastomer immersed in a racemic solvent. We have found considerably changes in the bandwidth of the reflectance for left- and right-copolarized light under the presence of the solvent which are susceptible to be detected experimentally. We have obtained a $R_{R R}$ bandwidth larger than that for an undistorted elastomer $(\alpha=0)$ for $\alpha=0.4$ and obliquely incidence for angles larger than $60^{\circ}$. Also in this value we have observed a thin band reflection for both polarizations $R_{R R}$ and $R_{L L}$ for oblique incidence.

The modifications of the transmittance and reflectance spectra during a preferentially absorbing process suggest to utilize the optical spectra as an indirect method to determine the concentration of preferentially absorbed molecules during a segregation process.

\section{Author details}

P. Castro-Garay

Depto de Fisica, Universidad de Sonora, Apdo Post. 1626, Hermosillo, Son. Mexico 83 000, México

Jesus Manzanares-Martinez

Depto. de Investigacion en Fisica, Universidad de Sonora, Hermosillo, Son., Mexico, Apdo P. 5-088, México 83190.

\section{References}

[1] A. Jakli, et al. One and two dimensional fluids: Properties of smetics, lamellar and columnar liquid crystals (Condensed Matter Physics). Taylor and Francis: Florida, EU, (2006).

[2] B. Singh. J. Phys. D: Appl. Phys., 40, 584 (2007).

[3] Y. Mao, M. Warner. Phys. Rev. Lett., 86, 5309, (2001).

[4] M. Warner, et al. Liquid Crystal Elastomers. Oxford Science Publications: Oxford, EU, (2007).

[5] M. Rivera, J. A. Reyes. Appl. Phys. Lett., 90, 023513, (2007).

[6] P. Cicuta, A. R. Tajbakhsh, E.M Terentjev. Phys. Rev. E., 70, 011703, (2004).

[7] S. Courty, A. R.Tajbakhsh, E. M. Terentjev. Phys. Rev. Lett., 91, 085503, (2003).

[8] Y. Mao,M. Warner. Phys. Rev. Lett., 84, 5335, (2000).

[9] A. Lakhtakia,W.S. Weiglhofer. Proc. R. Soc. Lond. A., 453, 93, (1997); correction: 454, 3275 (1998).

[10] N. Marcuvitz,J. Schwinger. J. Appl. Phys., 22, 806, (1951).

[11] P. Cicuta, A. R. Tajbakhsh. E. M. Terentjev. Phys. Rev. E., 65, 051704, (2002). 